normal controls. Patients underwent SPECT and neuropsychological examination at baseline; a second evaluation was conducted after a mean follow-up period of 18.7 months.

$A D$ developed in $16 \mathrm{MCl}$ patients, who were retrospectively diagnosed as having progressive $\mathrm{MCl}$ at baseline. The remaining $23 \mathrm{MCl}$ patients were diagnosed as having stable $\mathrm{MCl}$ at baseline. Patients with progressive $\mathrm{MCl}$ had significantly worse performance in neuropsychological tests at baseline than patients with stable $\mathrm{MCl}(P<0.05)$, and experienced further worsening in performance during followup. Baseline SPECT analysis of cerebral blood flow revealed hypoperfusion in the parietal lobe and hyperperfusion in the frontal lobe and cerebellum in progressive versus stable $\mathrm{MCl}$. During follow-up, blood flow decreased in the hippocampus, parahippocampus, parietal lobe and brainstem of patients with progressive $\mathrm{MCl}$, while perfusion increased in the medial frontal lobe, cingulate, and occipital lobe.

Multivariate analysis suggests a baseline diagnostic accuracy of $82 \%$ for SPECT and $84 \%$ for neuropsychological testing, and an accuracy of $92 \%$ for the combined techniques. The authors report that the level of perfusion in the parietal lobe is a sensitive marker for the development of dementia. They state that the combination of SPECT and neuropsychological tests can predict the development of $A D$ and monitor disease progression and the effects of therapy.

Original article Huang $C$ et al. (2006) Imaging markers of mild cognitive impairment: multivariate analysis of $\mathrm{CBF}$ SPECT. Neurobiol Aging [doi: 10.1016/

j.neurobiolaging.2006.05.017]

\section{Cognitive impairment is common in hemodialysis patients}

Patients on hemodialysis are at high risk of cognitive impairment because of their older age and high prevalence of stroke and cardiovascular risk factors. Murray et al. investigated cognitive function in a cohort of hemodialysis patients to identify factors that might be associated with cognitive impairment.

In total, 374 patients aged $\geq 55$ years who had been on maintenance hemodialysis for at least 2 months underwent cognitive tests that investigated memory, executive function and verbal fluency domains. A cognitive impairment algorithm, which required completion of tests in at least two of these domains, was used to classify subjects as having mild, moderate or severe cognitive impairment, or normal cognitive function.

Of the 338 patients who completed tests in at least two domains, 43 (13\%) were classified as having normal cognition, and 47 (14\%) as having mild, 122 (36\%) as having moderate and $126(37 \%)$ as having severe cognitive impairment; only $2.9 \%$ had a documented history of cognitive impairment. In adjusted models, equilibrated $K t / V>1.2$ (a marker of dialysis adequacy), history of stroke and $\leq 8$ years of education were associated with severe cognitive impairment $(P<0.05$ for all). Hemoglobin level $<110 \mathrm{~g} / \mathrm{l}$ was of borderline significance $(P<0.08)$. Risk of severe cognitive impairment was significantly higher in a random sample of 101 of the 338 hemodialysis patients than in 101 age-matched non-dialysis patients (adjusted odds ratio $3.54 ; P<0.02$ ).

The authors conclude that moderate to severe cognitive impairment is common in hemodialysis patients, and that improved detection of such impairment is needed. Further studies are required to ascertain whether cognitive impairment is caused solely by the underlying disease, or if it is worsened by hemodialysis.

Original article Murray AM et al. (2006) Cognitive impairment in hemodialysis patients is common. Neurology 67: $216-223$

\section{Site of infarct affects frequency and recovery from headache in lacunar stroke}

Headache is infrequent in acute lacunar infarction, but it might nevertheless have important clinical implications. Arboix et al. investigated whether the site of infarct affects the incidence of headache or neurological outcome, using data from 387 patients with acute lacunar infarction prospectively collected from a hospital-based stroke registry.

Forty-three (11.1\%) patients presented with headache within $72 \mathrm{~h}$ of stroke onset. Of these, $14.9 \%$ had deep brain gray matter or brainstem lacunar infarction, while only $8 \%$ had supratentorial white matter lacunar infarction. In patients with deep brain gray matter or brainstem 